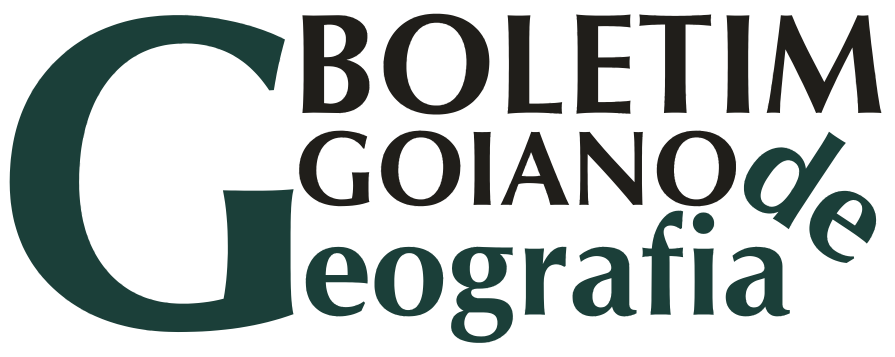

INSTITUTO DE ESTUDOS

SÓCIO-AMBIENTAIS/GEOGRAFIA

VOL. 23 - No 1 - JAN./ JUN. 2003

$\infty$
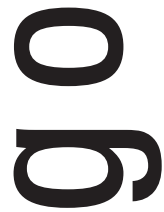

$\square$
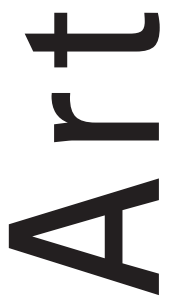


\title{
IDENTIFICAÇÃO DE ÁREAS PRIORITÁRIAS PARA CONSERVA- ÇÃO DA BIOGEODIVERSIDADE NO ESTADO DE GOIÁS
}

\author{
Pedro da Costa Novaes* \\ Laerte Guimarães Ferreira** \\ Rogério Dias ***
}

\section{Resumo}

A elevada diversidade biológica e a intensa pressão antrópica a que o cerrado vem sendo submetido fizeram desse bioma uma das 25 áreas mundialmente consideradas críticas para a conservação. Especificamente no Estado de Goiás, única unidade da federação totalmente inserida nos domínios do cerrado, aproximadamente $42,5 \%$ do território encontra-se recoberto por pastagens cultivadas, enquanto apenas $4,89 \%$ da área total do Estado representa as unidades de conservação federais, estaduais, municipais e particulares. Neste artigo, apresentamos o panorama atual das unidades de conservação no Estado e discutimos a identificação de áreas prioritárias para conservação, tendo em vista a estruturação e efetiva implementação do Sistema Estadual de Unidades de Conservação (SEUC).

Unitermos: Áreas Prioritárias para Conservação/ Conservação da Biodiversidade/ Cerrado.

\section{Introdução}

O cerrado brasileiro, presente em $25 \%$ do território nacional (cerca de 2 milhões de $\mathrm{km}^{2}$ ) e em 45\% da América do Sul, é considerado a savana mais rica do mundo no que concerne à biodiversidade (Eiten, 1993; Ratter et al., 1996). O cerrado abriga, segundo dados apresentados por Arruda (2001), mais de 10 mil espécies de plantas, das quais aproximadamente 4.400 são endêmicas, isto é, só ocorrem nas savanas brasileiras. Ainda segundo Arruda (2001), estudos recentes

* Secretaria de Meio Ambiente e Recursos Hídricos de Goiás. E-mail: pedro@semarh. goias.gov.br

** Instituto de Estudos Sócio-Ambientais (IESA)/UFG. E-mail: laerte@iesa.ufg.br ***Agência Goiana do Meio Ambiente (AGMA) - Consultor. E-mail: cerrado@tba. com.br 
NOVAES, Pedro da Costa et al. Identificação de áreas prioritárias para conservação da biodiversidade do estado de Goiás. Boletim Goiano de Geografia, 23 (1): 41 - 58, jan./jun. 2003

contabilizam 837 espécies de pássaros, 67 gêneros de mamíferos (abrangendo 161 espécies, 19 endêmicas), 150 espécies de anfíbios (45 endêmicas) e 120 de répteis (45 endêmicas). Apenas no Distrito Federal, há 90 espécies de cupins, mais de 1.000 espécies de borboletas e 500 espécies de abelhas e vespas.

Graças a esse elevado valor quanto à biodiversidade, o cerrado brasileiro foi considerado um dos 25 hotspots de biodiversidade no planeta (Myers et al., 2000). O cerrado desempenha ainda um importante papel no balanço de energia, água e carbono da região, atuando como um sumidouro de gases de efeito estufa, tais como o CO, $\mathrm{CO}_{2}$ e $\mathrm{CH}_{4}$ (Monteiro, 1995; Silva, 1999).

Em contrapartida, devido ao baixo custo de aquisição de terras, à topografia predominantemente plana, à construção de Brasília e à implantação, por parte do governo federal, de programas de desenvolvimento como o POLOCENTRO e o PRODECER, o cerrado passou a constituir o ecossistema brasileiro que tem sofrido maior pressão no tocante ao uso e à ocupação do solo, notadamente em Goiás e no Mato Grosso do Sul, importantes produtores de carne bovina, ou em Mato Grosso, com extensos cultivos de soja e milho. Estima-se que cerca de 40\% dos 208 milhões de hectares de cerrado contínuo já tenham sido convertidos em pastagens cultivadas, áreas agrícolas e outros tipos de uso (Mantovani \& Pereira, 1998; Sano et al., 2001).

Especificamente no Estado de Goiás - única unidade da federação totalmente inserida nos domínios do cerrado e caracterizada por um sistema natural que abriga parte considerável das nascentes das bacias hidrográficas de São Francisco, Araguaia/Tocantins e Paraná - aproximadamente 42,5\% do território encontra-se recoberto por pastagens cultivadas (Sano et al., 2001). Por sua vez as unidades de conservação federais, estaduais, municipais e particulares totalizam apenas 4,89\% do território goiano (Galinkin, 2001), índice muito inferior ao mínimo (10\%) considerado necessário pela maioria dos especialistas. Neste sentido, é imprescindível que novas áreas, ambientalmente sensíveis e/ou características, sejam preservadas por lei, possibilitando assim a conservação e preservação do ativo ambiental e da biodiversidade do Estado.

Este artigo discute a identificação de áreas prioritárias para conservação, tendo em vista a estruturação e efetiva implementação do Sistema Estadual de Unidades de Conservação (SEUC). Especificamente, abordamos o arcabouço metodológico e conceitual do projeto Identificação de Áreas Prioritárias para 
NOVAES, Pedro da Costa et al. Identificação de áreas prioritárias para conservação da biodiversidade do estado de Goiás. Boletim Goiano de Geografia, 23 (1): 41 - 58, jan./jun. 2003

Conservação no Estado de Goiás. Esse projeto, ora em curso e com término previsto para agosto de 2004, insere-se no contexto do componente ambiental do Programa de Gerenciamento Rodoviário - objeto de empréstimo contratado pelo Estado de Goiás no Banco Internacional para Reconstrução e Desenvolvimento (BIRD) -, haja vista a necessidade de se adequar o gerenciamento rodoviário aos critérios de desenvolvimento sustentável.

\section{Unidades de conservação no Estado de Goiás: panorama atual}

A exemplo do que ocorre em outros estados e países, a criação de unidades de conservação em Goiás tem seguido critérios muito mais políticos e econômicos do que razões de cunho científico que permitam uma adequada proteção da biodiversidade. A maioria dessas áreas é criada em função de seu valor cênico ou de outras questões contingenciais, como a existência de terras devolutas ou que possam ser adquiridas a baixos preços, o que acaba resultando em recortes territoriais inapropriados e hábitats inadequados para a proteção da biodiversidade.

Um exemplo dessa realidade foi a criação, em 1970, do Parque Estadual da Serra de Caldas, a mais antiga unidade de conservação no Estado de Goiás. Essa unidade, com área de 12.315,36 ha e situada nos municípios de Caldas Novas e Rio Quente, protege o maciço geológico que lhe dá nome, sem se estender aos ecossistemas contíguos associados.

A criação das demais unidades de proteção integral ao longo dos anos, a despeito do significativo valor quanto à biodiversidade de várias delas, seguiu, em geral, critérios ora paisagísticos, ora econômicos.

O Parque Estadual de Terra Ronca, em que pese seu inquestionável valor para a conservação da biodiversidade, conforme atesta mesmo sua inclusão entre os polígonos considerados prioritários para conservação, pelo Programa Nacional de Biodiversidade, ${ }^{1}$ tem sua criação devida essencialmente a seu complexo espeleológico associado a rochas carbonáticas do Grupo Bambuí. Sua delimitação, ainda que calcada em levantamentos técnicos, é considerada insuficiente pelo mesmo relatório do PROBIO que sugere sua ampliação para

1 http://www.mma.gov.br/biodiversidade/cobio.html 
NOVAES, Pedro da Costa et al. Identificação de áreas prioritárias para conservação da biodiversidade do estado de Goiás. Boletim Goiano de Geografia, 23 (1): 41 - 58, jan./jun. 2003

pelo menos oitenta mil hectares.

O mesmo vale para o Parque Estadual da Serra dos Pirineus, criado em 1987 para a proteção do conjunto paisagístico associado ao maciço quartzítico nos municípios de Pirenópolis, Corumbá de Goiás e Cocalzinho.

O Parque Ecológico Altamiro de Moura Pacheco, que protege um valioso remanescente de Floresta Atlântica do Interior no entorno de Goiânia, deve sua criação à boa vontade e ao idealismo do proprietário da área, que o nome da unidade de conservação homenageia e que a vendeu a preços abaixo do preço de mercado ao governo do Estado.

O Parque Estadual Telma Ortegal, no município de Abadia de Goiás, representa uma inadequação da categoria de unidade de conservação ao tipo de área que abrange e aos propósitos da própria unidade de conservação. Trata-se da área no entorno do depósito dos rejeitos radiativos do acidente com césio137 em Goiânia, destinada a salvaguardar esses rejeitos, assegurar a proteção da população do entorno e servir como espaço de educação ambiental. Além de relativamente reduzida, 166 ha, a área possui pouca ou nenhuma cobertura vegetal. Seria conveniente, em nosso entendimento, reenquadrar essa unidade de conservação, transformando-a em área de proteção ambiental (APA) ou área de relevante interesse ecológico (ARIE).

Mais recentemente foi criado, através do Decreto $\mathrm{n}^{\circ} 5.568$, de 18 de março de 2002, o Parque Estadual de Paraúna, essencialmente em função do conjunto paisagístico associado aos relevos tabuliformes e ruiniformes dos arenitos da formação aquidauana na Bacia Sedimentar do Paraná. Ainda mais recente é o Parque Estadual do Araguaia, situado no município de São Miguel do Araguaia e criado através do Decreto $\mathrm{n}^{\circ} 5.631$, de 2 de agosto de 2002. A instituição desse parque se deve às medidas de compensação ambiental do projeto de irrigação Luiz Alves, localizado em área contígua ao parque.

Dos exemplos acima, fica também evidente o avanço na criação e implantação de unidades de conservação, principalmente a partir de 1997, quando da criação da Diretoria de Unidades de Conservação na antiga Fundação Estadual de Meio Ambiente (FEMAGO), atual Agência Goiana de Meio Ambiente (AGMA). De fato, no Estado de Goiás, o percentual de território sob proteção no interior de unidades de conservação sofreu, nos últimos quatro anos, um aumento significativo. Segundo Galinkin (2001), em 1998, as unidades de conservação federais, estaduais e municipais e as Reservas Particulares do Patrimônio Na- 
NOVAES, Pedro da Costa et al. Identificação de áreas prioritárias para conservação da biodiversidade do estado de Goiás. Boletim Goiano de Geografia, 23 (1): 41 - 58, jan./jun. 2003

tural (RPPNs) somavam um total de 323.547,2 ha. Três anos depois, no final de 2001, esse valor aumentara para 1.672.228,1 ha, ${ }^{2}$ o que corresponde a $4,89 \%$ do território de Goiás - um aumento de 416,8\%. Consideradas apenas as unidades de conservação estaduais, passou-se, no mesmo período, de $0,24 \%$ do território estadual sob proteção legal para 3,2\%, num incremento de $1.309 \%$ na área protegida. A distribuição espacial, o grupo (unidades de proteção integral ou de uso sustentável) e a jurisdição (particular, municipal, estadual e federal) das unidades de conservação no Estado de Goiás são apresentados na Figura 1 e sintetizados na Tabela 1 .

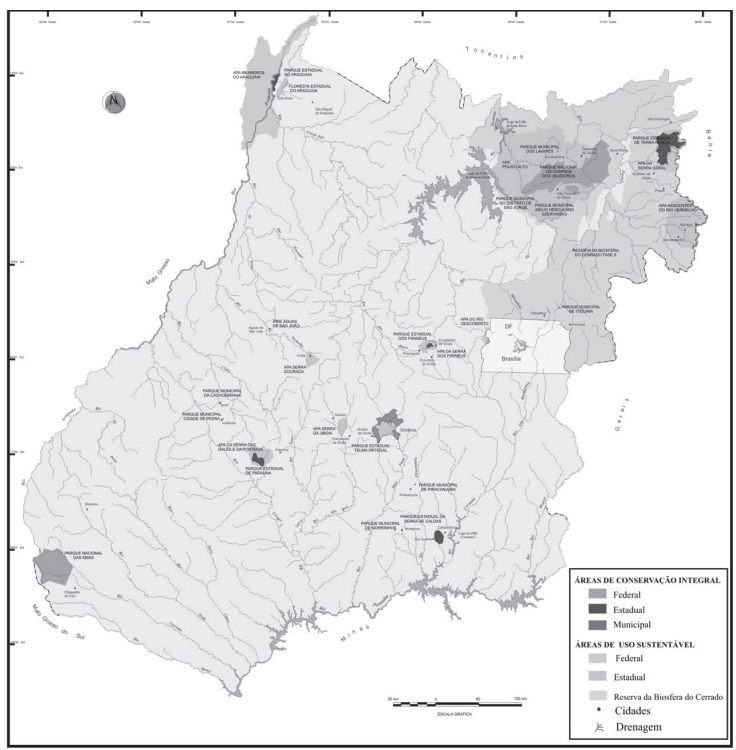

Figura 1 - Unidades de conservação no Estado de Goiás, organizadas em grupo (proteção integral e uso sustentável) e em jurisdição (federal, estadual e municipal)

Fonte: Goiás (2002)

2 Os dados incluem apenas as unidades de conservação criadas até o final de 2001. Além dessas, deve-se considerar a criação, em 2002, das seguintes unidades: Parque Estadual de Paraúna, APA Serra das Galés, APA João Leite (as três com área total ainda pendente de definição), Parque Estadual do Araguaia (4.611,8 ha), Floresta Estadual do Araguaia (8.202,81 ha) e APA Federal do Planalto Central, que abrange 
NOVAES, Pedro da Costa et al. Identificação de áreas prioritárias para conservação da biodiversidade do estado de Goiás. Boletim Goiano de Geografia, 23 (1): 41 - 58, jan./jun. 2003

Tabela 1 - Unidades de Conservação do Estado de Goiás (2001)

\begin{tabular}{|c|c|c|c|c|c|c|c|c|c|}
\hline \multirow{2}{*}{$\begin{array}{l}\text { Unidades de } \\
\text { Conservação }\end{array}$} & \multicolumn{2}{|c|}{$\begin{array}{c}\text { Bacia do } \\
\text { Paranaíba }\end{array}$} & \multicolumn{2}{|c|}{$\begin{array}{r}\text { Bacia do } \\
\text { Araguaia }\end{array}$} & \multicolumn{2}{|c|}{$\begin{array}{l}\text { Bacia do } \\
\text { Tocantins }\end{array}$} & \multicolumn{3}{|c|}{ Total } \\
\hline & Qt. & $\begin{array}{c}\text { Área em } \\
\text { Goiás }\end{array}$ & Qt. & $\begin{array}{c}\text { Área em } \\
\text { Goiás }\end{array}$ & Qt. & $\begin{array}{c}\text { Área em } \\
\text { Goiás }\end{array}$ & Qt. & $\begin{array}{c}\text { Área em } \\
\text { Goiás }\end{array}$ & $\begin{array}{l}\% \text { do } \\
\text { território } \\
\text { estadual }\end{array}$ \\
\hline FEDERAL & 2 & $130.256,5$ & 1 & 35.700 & 3 & $416.546,4$ & 6 & 582.503 & 1,71 \\
\hline Proteção Integral & 1 & 129.790 & - & - & 1 & 236.570 & 2 & 366.360 & 1,07 \\
\hline Uso Sustentável & 1 & 466,6 & 1 & 35.700 & 2 & $179.976,4$ & 4 & 216.143 & 0,63 \\
\hline ESTADUAL & 4 & $37.372,1$ & 2 & $16.877,5$ & 6 & $1.011 .799,9$ & 12 & $1.066 .049,6$ & 3,12 \\
\hline Proteção Integral & 3 & $15.621,1$ & - & - & 3 & $59.833,3$ & 6 & $75.454,4$ & 0,22 \\
\hline Uso Sustentável & 1 & 21.751 & 2 & $16.877,5$ & 3 & $951.966,7$ & 6 & $990.595,2$ & 2,90 \\
\hline MUNICIPAL & 7 & $3.105,5$ & 2 & 123,3 & 4 & 144,6 & 12 & $3.398,4$ & 0,00 \\
\hline Proteção Integral & 7 & $3.105,5$ & 2 & 123,3 & 4 & 144,6 & 12 & $3.398,4$ & 0,00 \\
\hline RPPN & 17 & $4.317,3$ & 3 & 4.059 & 21 & $11.925,8$ & 41 & $20.302,1$ & 0,06 \\
\hline TOTAL & 30 & $175.051,5$ & 8 & $56.759,8$ & 34 & $1.440 .416,8$ & 72 & $1.672 .228,1$ & 4,89 \\
\hline
\end{tabular}

A maior parte desse aumento se deu, entretanto, através da criação de unidades de conservação do grupo de uso sustentável, especialmente as APAs. Apenas a APA de Pouso Alto, no nordeste do Estado, criada no ano de 2000, com 872 mil hectares, responde por quase $50 \%$ da área protegida no Estado de Goiás. A ela somam-se as APAs Serra da Jibóia, nos municípios de Nazário e Palmeiras de Goiás, Serra Dourada, nos municípios de Goiás e Mossâmedes, Serra Geral, no entorno do Parque Estadual de Terra Ronca, dos Pirineus, no entorno do Parque Estadual da Serra dos Pirineus, do João Leite, que abrange a bacia hidrográfica de mesmo nome, e da Serra das Galés, no município de Paraúna.

Em relação às RPPNs e às unidades de conservação de proteção integral, nas três esferas de governo, tem-se apenas $1,35 \%$ do território estadual inserido em unidades de conservação com um grau relativamente satisfatório de garantias de preservação.

Da mesma forma, observa-se ainda (Tabela 1), no total das unidades de conservação, uma significativa concentração na localização da superfície 
NOVAES, Pedro da Costa et al. Identificação de áreas prioritárias para conservação da biodiversidade do estado de Goiás. Boletim Goiano de Geografia, 23 (1): 41 - 58, jan./jun. 2003

territorial protegida. Na Bacia do Tocantins, situam-se $86 \%$ dessas áreas contra $3,4 \%$ na Bacia do Araguaia e 10,6\% na Bacia do Paranaíba. Quando consideramos apenas as unidades de conservação de proteção integral e as RPPNs, esses percentuais são de $68 \%$ na Bacia do Tocantins, $0,02 \%$ na Bacia do Araguaia e $31,9 \%$ na Bacia do Paranaíba.

Essa baixa representatividade do território e de paisagens é ainda constatada quando da análise da distribuição das unidades de conservação do Estado em relação às ecorregiões existentes. O Estado de Goiás conta com duas ecorregiões, cerrado e Florestas do Interior do Paraná/Paranaíba, sendo que a segunda é representada apenas nos 3.139,77 ha do Parque Ecológico Altamiro de Moura Pacheco.

Ainda que macrobacias hidrográficas não representem necessariamente recortes territoriais adequados para se pensar a representatividade dos distintos padrões de biodiversidade no conjunto das unidades de conservação, essa análise dá uma idéia das lacunas a serem sanadas por uma política estadual de biodiversidade para que se protejam adequadamente os ecossistemas. É exatamente a necessidade de assegurar essa representatividade dos padrões de biodiversidade presentes no território do Estado ou do país que tem levado à institucionalização de sistemas - federais e estaduais - de unidades de conservação, tendo em vista fazer que esses espaços protegidos sejam criados e geridos com base em uma perspectiva integrada e de conjunto.

Neste sentido, um significativo avanço institucional no Estado de Goiás se deu com a aprovação da Lei ${ }^{\circ} 14.247$, de 29 de julho de 2002, relativa ao SEUC. Essa lei consolidou e adaptou, na esfera estadual, diretrizes e instrumentos para a criação e gestão de tais unidades em consonância com a Lei do Sistema Nacional de Unidades de Conservação (Lei Federal n 9.985, de 18 de julho de 2000).

\section{Priorização de áreas para conservação: iniciativas e abordagens}

A identificação de áreas prioritárias para conservação tem sido objeto de inúmeros estudos e projetos pilotos nos distintos biomas brasileiros (Dias, 2002; Jablonski, 2002; Maury, 2002; Silva, 2002; Capobianco, 2001; Pinto, 2000; Costa, 1998). Especificamente em relação ao bioma cerrado, vale destacar o projeto Ações Prioritárias para a Conservação da Biodiversidade do Cerrado e 
NOVAES, Pedro da Costa et al. Identificação de áreas prioritárias para conservação da biodiversidade do estado de Goiás. Boletim Goiano de Geografia, 23 (1): 41 - 58, jan./jun. 2003

Pantanal, que identificou 87 áreas prioritárias para conservação nesses dois biomas, sendo 15 delas no Estado de Goiás (Cavalcanti, 1999).

A identificação e delimitação dessas áreas, ainda que baseada em parâmetros abióticos (grandes domínios de solos, clima e relevo) e na urgência de ações de conservação (determinada a partir das pressões demográficas, da vulnerabilidade das áreas naturais às atividades econômicas, da expansão urbana e da infra-estrutura planejada), fundamentou-se principalmente na distribuição dos elementos da biota, enfocando em particular critérios como riqueza de espécies, grau de endemismo, ocorrência de espécies raras ou ameaçadas, espécies migratórias, além de aspectos culturais e econômicos associados às espécies e grandes paisagens.

Este estudo, ainda que revestido de grande importância e mérito, por ter sido a primeira e única iniciativa de caráter científico voltada à identificação de áreas prioritárias para conservação no bioma cerrado, é alvo de duas críticas severas quanto ao uso efetivo dos seus resultados. A primeira diz respeito à escala de análise, considerada muito superficial, mesmo no âmbito mesorregional a que o trabalho se propôs. Um exemplo nesse sentido é o mapa de integridade da cobertura vegetal do cerrado, cuja elaboração ficou a cargo do Instituto Nacional de Pesquisas Espaciais (Mantovani \& Pereira, 1998). Esse mapa, ainda que à escala de 1:250.000, teve por base a interpretação de imagens Landsat 5 obtidas entre 1987 e 1993, e compreende apenas quatro classes de cobertura e uso da terra (1 - não-cerrado; 2- cerrado não antropizado; 3- cerrado antropizado; 4- cerrado fortemente antropizado). Essa restrição resultou em informações defasadas e incompatíveis com a complexidade e dinâmica de ocupação do cerrado, além de ter limitado a identificação de manchas contínuas de vegetação nativa às áreas superiores a cem mil hectares.

A segunda e principal crítica à abordagem metodológica utilizada no estudo em questão diz respeito à ênfase no componente biótico, e em particular na distribuição de espécies, alegando conhecimento insuficiente e desigual sobre padrões de distribuição, riqueza e diversidade de muitos dos grupos biológicos. Assim, o mapa resultante dos vários cruzamentos temáticos, em vez de sintetizar e indicar áreas de alta prioridade quanto à conservação, reflete em princípio o estado-da-arte do conhecimento sobre a biodiversidade (Nelson et al., 1990). Uma alternativa à identificação de áreas com base na distribuição de espé- 
NOVAES, Pedro da Costa et al. Identificação de áreas prioritárias para conservação da biodiversidade do estado de Goiás. Boletim Goiano de Geografia, 23 (1): 41 - 58, jan./jun. 2003

cies é aquela que priveligia a diversidade e representatividade de ecossistemas, tendo por base a hipótese de que as variações na paisagem correspondem ao conjunto de processos ecológicos em seus distintos graus de complexidade. Em outras palavras, a manutenção das condições ecológicas encontradas em uma dada área garante a preservação da maior parte das espécies nela existentes.

Uma crítica comum a esta abordagem também diz respeito à escala superficial de análise. Um outro fator limitante concerne à própria definição das unidades de paisagem (biogeográficas), na medida em que o limite destas depende das variáveis e critérios utilizados. Por exemplo, Silva (1998), em um estudo para o estabelecimento de áreas prioritárias na Amazônia Legal, considerou como unidades biogeográficas as regiões interfluviais do bioma Amazônia, ao contrário de Fearnside \& Ferraz (1995), que, em estudo semelhante, consideraram como unidades de análise os Estados da Amazônia Legal.

À dificuldade em se definir e estabelecer unidades biogeográficas contrapõe-se o conceito de ecorregiões, que correspondem a um conjunto de comunidades naturais, geograficamente distintas, que compartilham a maioria das suas espécies, dinâmicas e processos ecológicos, e condições ambientais similares, nas quais as interações ecológicas são críticas para sua sobrevivência a longo prazo (Veloso et al., 2002; Dinerstein et al., 1995). Segundo Ferreira (2002), o uso da ecorregião como unidade de análise da paisagem possibilita um significativo avanço no que diz respeito à conservação da biodiversidade, em função da maior possibilidade de incorporação de escalas (biomas ${ }^{\circledR}$ ecorregiões ${ }^{\circledR}$ unidades de paisagem), de processos ecológicos e principalmente dos processos socioeconômicos na análise de representatividade das áreas prioritárias para a conservação.

\section{O projeto "Identificação de Áreas Prioritárias para Conservação no Estado de Goiás": proposta metodológica}

O projeto "Identificação de Áreas Prioritárias para Conservação no Estado de Goiás" fundamenta-se na ecologia de paisagens, mais precisamente na identificação e delimitação de unidades de paisagem (ecorregiões) à escala de 1:250.000, na análise da representatividade dessas paisagens nas unidades de conservação já implementadas (análise de lacunas), bem como na utilização de modelos logísticos que envolvem tanto variáveis socioeconômicas quanto 
ecológicas.

Regressões logísticas são modelos empíricos caracterizados por sua simplicidade e eficiência em fazer predições (previsão de cenários), ainda que limitados quanto a análises dinâmicas (evolução da paisagem) (Câmara \& Pedrosa, 2002). O uso desses modelos resulta em mapas de probabilidade, como, por exemplo, a probabilidade de desmatamentos em função da proximidade de estradas. Da mesma forma, por meio de pesos diferenciados e arbitrários, podem-se gerar mapas que indiquem a probabilidade de uma nova unidade de conservação situar-se nas proximidades de centros urbanos ou de futuras obras de infra-estrutura (pesos negativos e baixas probabilidades) ou nas proximidades de unidades de conservação já existentes e de outros remanescentes de vegetação (pesos positivos e altas probabilidades).

Quanto à identificação e delimitação de unidades representativas da paisagem, estudos semelhantes, como, por exemplo, o conduzido por Ferreira (2002) para a definição de áreas prioritárias para conservação no bioma Amazônia, propõem o cruzamento temático entre as classes de solos, como indicador de diversidade beta (diversidade de paisagens), e entre as fisionomias de vegetação, como indicador da diversidade florística. No que se refere ao projeto em questão, a diversidade de paisagens está sendo definida principalmente com base em domínios geomorfológicos, haja vista a inexistência no Estado de informações de solos atualizadas e em escala compatível (1:250.000). Além disso, deve-se ressaltar que a escala de trabalho adotada, as geoformas e a cobertura vegetal são os elementos mais facilmente identificáveis na paisagem, sendo esses os principais condicionadores dos demais fatores ambientais, ao mesmo tempo em que possibilitam o entendimento das interações entre os elementos bióticos e seus suportes abióticos.

Especificamente, o projeto proposto e ora em execução baseia-se em um conjunto de mapeamentos/levantamentos temáticos obtidos por meio da interpretação e análise de imagens Landsat 7, passagens de 2001 e 2002, compreendendo um intervalo temporal não superior a quatro meses, dados de campo e mapas de vegetação, solo e geomorfologia do Projeto RADAMBRASIL, além de uma extensa e atual base de dados recentemente disponibilizada em formato digital. ${ }^{3}$ A base de dados a ser gerada, bem como as especificidades e características dos vários mapeamentos e levantamentos temáticos que a 
NOVAES, Pedro da Costa et al. Identificação de áreas prioritárias para conservação da biodiversidade do estado de Goiás. Boletim Goiano de Geografia, 23 (1): 41 - 58, jan./jun. 2003

compõem, é detalhada na Tabela 2.

Da integração dessas informações através de métodos de análise e modelagem espacial resultará o mapa-síntese com a indicação das áreas prioritárias para a criação de novas unidades de conservação no Estado de Goiás.

Tabela 2 - Base de Dados em Elaboração com vistas à Identificação de Áreas Prioritárias à Conservação no Estado de Goiás

\begin{tabular}{|c|c|c|c|}
\hline Categoria & Produtos & \multicolumn{2}{|c|}{ Características e Especificações } \\
\hline \multirow{3}{*}{ Mapas-Base } & Base Cartográfica & \multicolumn{2}{|c|}{ Atualização da Iifra-estrutura existente (e.g. malha viária) e planejada } \\
\hline & Declividade & \multicolumn{2}{|l|}{ Gradiente e aspecto } \\
\hline & Hipsometria & \multicolumn{2}{|c|}{ Conforrme eqüidistâncias das curvas de nível e quebras de relevo } \\
\hline \multirow{2}{*}{$\begin{array}{l}\text { Mapas de Cobertura } \\
\text { Vegetal e Formas de Uso }\end{array}$} & Regiões Fitoecológicas & \multirow{2}{*}{\multicolumn{2}{|c|}{ Metodologia IBGE }} \\
\hline & Uso da Terra & & \\
\hline \multirow{3}{*}{$\begin{array}{l}\text { Mapas de Estrutura e } \\
\text { Organização da Paisagem }\end{array}$} & Geomorfologia & $\begin{array}{l}\text { Êfase na morfologia, morfometria e } \\
\text { gênese }\end{array}$ & $\begin{array}{l}\text { Conforme ZEEs de } \\
\text { Rondônia e Mato Grosso }\end{array}$ \\
\hline & Unidades de Paisagem & \multicolumn{2}{|c|}{$\begin{array}{l}\text { Conforme regiões fitoecológicas, compartimentação geomorfológica, } \\
\text { hipsometria e elementos do relevo }\end{array}$} \\
\hline & Vulnerabilidade Natural & $\begin{array}{l}\text { Conforme relação entre morfogênese e } \\
\text { pedogênese }\end{array}$ & $\begin{array}{l}\text { Metodologia de Crepani et } \\
\text { al. (1998) }\end{array}$ \\
\hline \multirow{3}{*}{ Mapas Acessórios } & $\begin{array}{l}\text { Unidades de Conservação } \\
\text { e Terras Indígenas }\end{array}$ & \multicolumn{2}{|l|}{ Federal, estadual e municipal } \\
\hline & $\begin{array}{l}\text { Patrimônio Histórico, } \\
\text { Arqueológico e } \\
\text { Espeleológico }\end{array}$ & \multicolumn{2}{|c|}{ Conforme levantamentos IBAMA, IPHAN eCECAV } \\
\hline & $\begin{array}{l}\text { Conhecimento sobre a } \\
\text { Biodiversidade }\end{array}$ & Inventários científicos & $\begin{array}{l}\text { Referência locacional: } \\
\text { Municícipios e UCs }\end{array}$ \\
\hline
\end{tabular}

Em um primeiro momento, e tendo em vista a identificação preliminar de áreas potenciais para conservação, estão sendo considerados os seguintes critérios e parâmetros, conforme a ordem a seguir:

3 SIG Goiás, elaborado pela Agência Goiana de Desenvolvimento Industrial e Mineral (AGIM), o qual inclui, entre outros, a base cartográfica do Estado, 1:250.000, o mapa geológico do Estado, compilado à escala de 1:500.000 pelo Serviço Geológico Nacional (CPRM), e diferentes diagnósticos ambientais realizados pela Fundação IBGE (exempli gratia, Zoneamento Econômico Ecológico da Região Nordeste do Estado). 
a) a diversidade de paisagens (ecorregiões), conforme evidenciado através dos mapas de regiões fitoecológicas e de unidades de paisagem;

b) a dimensão dos remanescentes de vegetação natural, medida pela diversidade de espécies e pelo seu tamanho populacional;

c) a proximidade entre os remanescentes vegetais, assumindo que hábitats contíguos e conectados são preferíveis àqueles fragmentados;

d) a preferência por blocos de remanescentes vegetais que incluam nascentes de rios e córregos ou microbacias hidrográficas;

e) a distância dos remanescentes vegetais em relação aos centros urbanos, pólos agroindustriais e atividades agropecuárias, assumindo que hábitats não cortados por estradas ou de difícil acesso são preferíveis e mais adequados à conservação;

f) a coincidência ou proximidade dos remanescentes vegetais com as áreas definidas pelo PROBIO, do Ministério do Meio Ambiente, como “Corredores Ecológicos” e "Áreas Prioritárias para a Conservação do Cerrado" (Cavalcanti, 1999);

g) a representatividade das paisagens nos remanescentes de vegetação identificados, isto é, a preferência por paisagens ainda pouco representadas nas unidades de conservação já implementadas.

Em uma segunda etapa, deverão ser determinados o grau de prioridade, em uma escala crescente de um a quatro, a categorização dessas áreas potenciais em unidades de conservação de proteção integral ou uso sustentável, e ainda as áreas com carência de estudos. Para tanto, serão necessários o refinamento e a realização de novos cruzamentos temáticos, bem como a definição de regiões buffer para cada estrada, tendo em vista o impacto que essas infra-estruturas têm sobre a cobertura vegetal e conseqüentemente sobre os hábitats (Nepstad et al., 2001; Laurance et al., 2001). Esses cruzamentos, por sua vez, terão por base diferentes equações logísticas, segundo um conjunto de variáveis favoráveis e desfavoráveis.

Exemplos de variáveis favoráveis incluirão, entre outros, a existência ou proximidade de patrimônio histórico, arqueológico e espeleológico, o grau de vulnerabilidade da paisagem, em uma escala crescente de valores, 
NOVAES, Pedro da Costa et al. Identificação de áreas prioritárias para conservação da biodiversidade do estado de Goiás. Boletim Goiano de Geografia, 23 (1): 41 - 58, jan./jun. 2003

e a ocorrência de estudos que sugiram a riqueza de espécies e a existência de espécies indicadoras. Por sua vez, as variáveis desfavoráveis deverão ser atribuídas às áreas desmatadas ou cortadas por estradas, aos projetos de infraestrutura etc.

\section{Considerações finais}

O cerrado é uma das 25 áreas mundialmente consideradas críticas quanto à necessidade de conservação, em virtude da elevada diversidade biológica e da intensa pressão antrópica a que esse bioma vem sendo submetido. Contudo, poucas são as áreas efetivamente protegidas (aproximadamente $1,7 \%$ ) e estas apresentam ainda o inconveniente de estarem concentradas em poucas regiões, cujo número é incompatível com o acentuado endemismo e com a extensa distribuição geográfica de espécies nesse bioma.

Contrapondo-se a essa realidade, o Estado de Goiás vem se destacando na criação e implantação de novas unidades de conservação, que hoje totalizam aproximadamente $4,89 \%$ do seu território. Especificamente em relação às unidades de conservação estaduais, estas representam atualmente 3,2\% da área do Estado, um aumento significativo quando se compara esse percentual aos $0,24 \%$ do território sob proteção legal estadual quatro anos atrás.

Contudo, deve-se ressaltar que a maior parte da área conservada do Estado encontra-se na forma de APAs que não se encontram zoneadas nem estão sob qualquer medida efetivada de restrição ao uso do solo. Tampouco nenhuma delas possui seu conselho consultivo implantado.

Ainda que a eficácia das APAs no tocante à proteção efetiva da biodiversidade seja em geral baixa, estas são de grande importância no âmbito de uma rede de áreas protegidas para o Estado. Assim, as APAs devem ser geridas como recortes territoriais apropriados para a implementação de modelos sustentáveis de desenvolvimento e embasados no aproveitamento das potencialidades naturais e sociais de seus territórios e populações. $\mathrm{O}$ foco dessas áreas deve ser o do desenvolvimento (sustentável) e não o da conservação por si só. Exemplos de propostas nesse sentido podem ser encontrados em Alves (2002), Quan \& Souza (2002), Carvalhêdo et al. (2002), Torres \& 
NOVAES, Pedro da Costa et al. Identificação de áreas prioritárias para conservação da biodiversidade do estado de Goiás. Boletim Goiano de Geografia, 23 (1): 41 - 58, jan.jun. 2003

Mesquita (2002), entre outros.

A falta de modelos de gestão adequados para essas unidades de uso sustentável é o reflexo direto da falta de uma política mais ampla e coerente, na esfera estadual, para a conservação da biodiversidade. Com respeito a esse tipo de iniciativa, deve-se destacar a aprovação da Lei n ${ }^{\circ}$. 14.247, de 29 de julho de 2002, que, ao criar o SEUC, estabelece as diretrizes e as bases jurídico-institucionais para a gestão e a consolidação das unidades de conservação, de proteção integral e de uso sustentável.

Da mesma forma, é imprescindível a agregação de conhecimentos e do desenvolvimento de uma nova cultura nas instituições estaduais como um todo. O projeto Identificação de Áreas Prioritárias para Conservação no Estado de Goiás, no âmbito do Programa de Gerenciamento Rodoviário do Estado, é certamente um passo decisivo neste sentido, na medida em que cria a possibilidade de integração entre os órgãos ambientais e de infra-estrutura. Esse projeto, cuja conclusão está prevista para agosto de 2004, representa de fato a primeira iniciativa quanto à utilização de critérios científicos para a definição de áreas prioritárias para conservação em Goiás. A sólida base de informações científicas a ser gerada (Tabela 2), inédita e só comparável em importância ao projeto RADAMBRASIL, servirá não apenas à manutenção da biodiversidade, mas também à gestão ambiental integrada do Estado. Em relação a essa base de dados, a ser disponibilizada integralmente na internet, é ainda importante enfatizar o seu formato aberto, que permitirá a constante atualização e agregação de novas informações.

Por fim, deve-se mencionar que essa base de dados constitui-se, em função de sua escala, de seus formatos e da natureza de suas informações, no mais importante subsídio ao futuro Zoneamento Econômico-Ecológico do Estado (ZEE). Em princípio, a manutenção da biodiversidade depende tanto de mecanismos de gestão ambiental quanto daqueles voltados à gestão territorial, como os ZEEs, os planos diretores territoriais e o gerenciamento de bacias hidrográficas.

\section{ABSTRACT \\ Identification of Priority Áreas for Bio-Geodiversity Conserva- tion in the State of Goiás}


NOVAES, Pedro da Costa et al. Identificação de áreas prioritárias para conservação da biodiversidade do estado de Goiás. Boletim Goiano de Geografia, 23 (1): 41 - 58, jan./jun. 2003

The large biological diversity and the intense anthropic pressure made the Cerrado biome one of the 25 world's biodiversity hotspots. Specifically concerning the State of Goiás, which is the only state in Brazil thoroughly within the Cerrado biome, approximately $45 \%$ of its area has already been aggressively converted into cultivated pastures. On the other hand, conservation units comprises only $4,89 \%$ of the State area. In this paper, we discuss the current scenario regarding conservation units in the State of Goiás. In addition, we also present the project "Identification of Priority Areas for Conservation", which is aimed the effective and operational implementation of a State level conservation unit system.

Key words: Priority Areas for Conservation, Biodiversity Conservation, Cerrado

\section{REFERÊNCIAS}

ALVES, S. M. de Brito. Gestão de áreas de proteção ambiental: um modelo em construção. In: CONGRESSO BRASILEIRO DE UNIDADES DE CONSERVAÇÃO, 3., 2002, Fortaleza. Anais... Fortaleza: Rede Nacional Pró-Unidades de Conservação, 2002. p. 259-267.

ARRUDA, M. B. (Org.). Ecossistemas brasileiros. Brasília: Edições IBAMA, 2001. 49 p.

CÂMARA, G.; PEDROSA, B. M. Geoprocessamento e geração de cenários para o ordenamento territorial. 2002. Disponível em: <http://www.zee.ma.gov.br>.

CAPOBIANCO, J. P. R. (Coord.) Avaliação e identificação de ações prioritárias para a conservação, utilização sustentável e repartição dos benefícios da biodiversidade na Amazônia Brasileira. Brasília, DF: ISA, IPAM, GTA, CI, IMAZON, ISPN, CE, GEF, Banco Mundial, WWF, NRF, USAID, ICCO, Gov. Amapá, CEFRH-AP, MCT/CnPq/FINEP, MMA/SBF, 2001. 144 p.

CARVALHÊDO, S. P. et al. Área de Proteção Ambiental do Pratigi: uma experiência inovadora de parcerias. In: CONGRESSO BRASILEIRO DE UNIDADES DE CONSERVAÇÃO, 3., 2002, Fortaleza. Anais... Fortaleza: Rede Nacional 
Pró-Unidades de Conservação, 2002. p. 251-258.

CAVALCANTI, R. (Coord.) 1999. Ações prioritárias para a conservação da biodiversidade do cerrado e Pantanal. Brasília, DF: Funatura, Biodiversitas, CI, UnB, GEF, Banco Mundial, MCT/CNPq, MMA/PROBIO, 1999. 26 p.

CAVALCANTI, R. B. Ações prioritárias para conservação da biodiversidade do Cerrado e do Pantanal. Mapa. Washington, DC: Conservation International, 1999.

COSTA, C. M. R. et al. Biodiversidade em Minas Gerais: um atlas para sua conservação. Belo Horizonte, MG: Fundação Biodiversitas, 1998. 94 p.

CREPANI, E.; MEDEIROS, J.; AZEVEDO, L.; HERNANDEZ, P. FLORENZANO, T.; DUARTE, V. \& FARIA, C. Sensoriamento remoto e geoprocessamento aplicados ao zoneamento ecológico-econômico. São José dos Campos: Ministério Ciência e Tecnologia/ INPE, 1998. 53p.

DIAS, B. F. S. (Coord.) PROBIO-Project for the Conservation and Sustainable Use of Brazilian Biological Diversity: activities report (1996-2002). Brasília, DF: MMA/SBF, 2002. 73 p.

DINERSTEIN, E. et al. A conservation assessment of the terrestrial ecoregions of Latin America and the Caribbean. The World Bank, Washington, DC, USA. (Basis for ecoregions of Latin America and the Caribbean), 1995.

EITEN, G. Cerrado's vegetation. In: PINTO, M. N. (Ed.) Cerrado: caracterização, ocupação e perspectivas. $2^{\text {nd }}$ ed. Brasília: Ed. UnB, 1993. p. 17-73.

FEARNSIDE, P. M. Potential impacts of climatic change on natural forests and forestry in Brazilian Amazônia. Forest Ecology and Management, n. 78, p. 51-70, 1995.

FEARNSIDE, P. M; FERRAZ, J. A conservation gap analysis of Brazil's Amazonian vegetation. Conservation Biology. Blackwell Science, v. 9, n. 5, p. 1134-1147, 1995.

FERREIRA, L. V. O uso de ecologia da paisagem na escolha de áreas prioritárias para a conservação da biodiversidade e análise de lacunas para a escolha de áreas prioritárias para a conservação da biodiversidade no bioma Amazônia: um instrumento de planejamento no Zoneamento Econômico-Ecológico. 2002. Disponível em: <http://www.zee.ma.gov.br>. 
NOVAES, Pedro da Costa et al. Identificação de áreas prioritárias para conservação da biodiversidade do estado de Goiás. Boletim Goiano de Geografia, 23 (1): 41 - 58, jan./jun. 2003

GALINKIN, M. (Ed.). Estado ambiental de Goiás 2001: atualização. Goiânia: Agência Ambiental de Goiás, 2001. 60 p.

JABLONSKI, S. (Coord.). Avaliação e ações prioritárias para a conservação da biodiversidade das zonas costeiras e marinha. Brasília, DF: Bio Rio, SECTAM-PA, SNE, IDEMA, SEMA-SP, FEPAM-RS, GEF, Banco Mundial, $\mathrm{MCT} / \mathrm{CNPq}, \mathrm{MMA} / \mathrm{SBF}, 2002.72 \mathrm{p}$.

LAURANCE, W. F. et al. The future of the Brazilian Amazon Science, v. 19, n. 291, p. 438-439, 2001.

MANTOVANI, J. E.; PEREIRA, A. Estimativa da integridade da cobertura de vegetação do cerrado através de dados Landsat - TM. In: SIMPÓSIO BRASILEIRO DE SENSORIAMENTO REMOTO, 9., 1998, Santos, SP. Versão em CD-ROM.

MAURY, C. M. (Org.). Biodiversidade brasileira: avaliação e identificação de áreas e ações prioritárias para a conservação, utilização sustentável e repartição de benefícios da biodiversidade brasileira. Brasília, DF: MMA/SBF, Programa Nacional de Conservação da Biodiversidade, 2002. 404 p. ( Série Biodiversidade n. 5).

MONTEIRO, J. M. G. Fluxo de CO, em um cerrado stricto sensu. $1995.61 \mathrm{f}$. Dissertação (Mestrado) - Universidade de Brasília, Brasília, 1995.

MYERS, N. et al. Biodiversity hotspots for conservation priorities. Nature, Londres, n. 403, p. 853-858, 2000.

NELSON, B.W. et al. Endemism centres, refugia and botanical collection density in Brazilian Amazônia. Nature, Londres, v. 345, n. 6.277, p. 714-716, 1990.

NEPSTAD, D. et al. Road paving, fire regime feedbacks, and the future of the Amazon forests. Forest Ecology and Management, v. 5524, p. 1-13, 2001.

PINTO, L. P. (Coord.). Avaliação e ações prioritárias para a conservação da biodiversidade da Mata Atlântica e Campos Sulinos. Brasília, DF: CI, SOS Mata Atlântica, Biodiversitas, IPE, SEMA-SP, IEF-MG, GEF, Banco Mundial, MCT/CNPq, MMA/SBF, 2000. 40 p.

QUAN, J. F.; SOUZA, M. L. C. Análise dos interessados para a área de proteção ambiental Litoral Norte da Bahia: uma ferramenta fundamental para a constru- 
ção da gestão participativa. In: CONGRESSO BRASILEIRO DE UNIDADES DE CONSERVAÇÃO, 3., 2002, Fortaleza. Anais... Fortaleza: Rede Nacional Pró-Unidades de Conservação, 2002. p. 167-176.

RATTER, J. A. et al. Analysis of the floristic composition of the Brazilian cerrado vegetation II: comparison of the woody vegetation of 98 áreas. Edinburgh Journal of Botanics, n. 53, p. 153-180, 1996.

SANO, E. E.; BARCELLOS, A. O.; BEZERRA, H. S. Assessing the spatial distribution of cultivated pastures in the Brazilian savanna. Pasturas Tropicales, v. 22, n. 3, p. 2-15, 2001.

SILVA, G. T. Fluxos de $\mathrm{CO}_{2}$ em um campo sujo submetido à queimada prescrita. 1999. 64 f. Dissertação (Mestrado em Ecologia) - Universidade de Brasília, Brasília, 1999.

SILVA, J. M. C. (Coord.). Avaliação e ações prioritárias para a conservação da biodiversidade da caatinga. Brasília, DF: UFPE, Biodiversitas, CI, Embrapa/Semi-Árido, GEF, Banco Mundial, MCT/CNPq, MMA/SBF, 2002. 36 p.

SILVA, J. M. C. Um método para o estabelecimento de áreas prioritárias para a conservação na Amazônia Legal, 1998. 18 p. Relatório técnico do WWF.

TORRES, L. M.; MESQUITA, C. A. B. Conselho Gestor da Área de Proteção Ambiental da Costa de Itacaré - Serra Grande: uma experiência de gestão participativa. In: CONGRESSO BRASILEIRO DE UNIDADES DE CONSERVAÇÃO, 3., 2002, Fortaleza. Anais... Fortaleza: Rede Nacional Pró-Unidades de Conservação, 2002. p. 278-288.

VELLOSO, A. L. et al. (Ed.). Ecorregiões propostas para o bioma caatinga. Recife, PE: PNE, TNC, 2002. 76 p. 